\title{
Combined Gate Replacement and Transmission Gate Based Logic Insertion Algorithm for Leakage Optimization in VLSI Circuits
}

\author{
V. Leela Rani \\ Associate Professor, ECE Department, GVP College of Engineering (Autonomous), Visakhapatnam, \\ Andhra Pradesh.
}

Dr. M. Madhavi Latha

Professor, ECE Department, JNTU College of Engineering, Hyderabad, Telangana

\begin{abstract}
Leakage power reduction is one of the key design goals in the present emerging trend of battery operated applications. Several researchers proposed numerous design techniques based on high threshold voltage devices to reduce power consumption. This needs additional process steps, which leads to increase in cost during the fabrication. In this paper, a novel approach called combined Gate Replacement and Transmission gate based logic insertion Algorithm is proposed. The proposed approach is well suited for circuits with more logic depth and the same is depicted through simulation results. Different benchmarks of ISCAS'89 are simulated to validate the proposed approach. Furthermore, comparative analysis of the proposed approach is performed with respect to Gate Replacement Algorithm. Simulation results explore that proposed approach could achieve $16.41 \%$ more leakage reduction compared to a conventional Gate Replacement technique. All benchmarks are simulated using HSPICE Tool based on BSIM3 transistor model parameters with $180 \mathrm{~nm}$ technology. Finally, the efficacy of the proposed approach in improving various metrics has been compared with present state-of-art methods.
\end{abstract}

Keywords: Leakage Power, Low Power, Transmission gate, Gate Replacement.

\section{Introduction}

Scaling of feature sizes has provided a remarkable advancement in VLSI industry. As technology scales down, thinner oxide layers, reduced threshold and supply voltages led to a significant increase in leakage currents. Many studies have proved that leakage power has come up to $40 \%$ of the total power dissipation [1]. Hence, reducing the leakage power has been a major challenge in recent years. Consequently, the need for circuit level and system-level power-efficient design techniques has grown considerably. Using threshold voltage variations and inserting sleep transistors, several design methods have been addressed formerly [2-5]. Though above mentioned techniques are good at reducing leakages, additional process steps are required during the fabrication to have high threshold devices. Input Vector Control (IVC) approach is one of the popular methods [6] which is independent of process parameters. IVC is based on a concept of stacking effect in a circuit $[7,8]$. Shortcoming of IVC technique is it does not suit for circuits with more logic depth. Gate Replacement technique is one of the effective methods to reduce leakage power [6]. In this paper, Combined Gate Replacement and Transmission Gate based logic insertion Algorithm is proposed to have better leakage reduction. Rest of the paper is organized as follows: preliminaries are presented in section 2, proposed work is introduced in section 3, Results and analysis are presented in a section 4 and conclusions are given in section 5 .

\section{Preliminaries}

This section presents a survey of efforts made by various researchers in reducing leakage using different reduction techniques.

\section{i. Input Vector Control (IVC) approach}

IVC is a well-known method of reducing leakage for efficient power management. Halter and Najm [9] introduced IVC approach. Leakage current dependency on input vector due to the stacking effect of transistors was analyzed in [7-10]. Leakage power calculations for all input combinations of a test circuit are to be measured to form a Look Up Table (LUT). TABLE.1 and TABLE. 2 present leakage values of two-input and three-input NAND gates. By exhaustive search in IVC method, test pattern which can give minimum leakage is determined among different input patterns [11]. Logic circuit is forced to this Minimum Leakage Vector (MLV) during sleep mode to reduce leakage in IVC approach [12], as shown in Fig.1. Estimating power by exhaustive simulations is infeasible for larger circuits. Alternatively, many researchers have used various Heuristic Algorithms to find a minimum leakage state. Linear search method is presented in [13] to determine MLV. Abdoul Rjoub et al. proposed Fast Input Vector Control Algorithm in [14] to search for MLV. Genetic Algorithm is used in $[15,16]$ to find the minimum leakage state.

TABLE.1. Leakage values of two input NAND gate

\begin{tabular}{|c|c|c|}
\hline S. No & State & Leakage power $(\mathrm{W})$ \\
\hline 1 & 00 & $154.77 \mathrm{f}$ \\
\hline 2 & 01 & $5.73 \mathrm{p}$ \\
\hline 3 & 10 & $5.44 \mathrm{p}$ \\
\hline 4 & 11 & $10.48 \mathrm{p}$ \\
\hline
\end{tabular}


TABLE.2. Leakage values of three input NAND gate

\begin{tabular}{|c|c|c|}
\hline S.No & State & Leakage power $(\mathrm{W})$ \\
\hline 1 & 000 & $36.85 \mathrm{f}$ \\
\hline 2 & 001 & $154.77 \mathrm{f}$ \\
\hline 3 & 010 & $92.79 \mathrm{f}$ \\
\hline 4 & 011 & $5.73 \mathrm{p}$ \\
\hline 5 & 100 & $955.96 \mathrm{f}$ \\
\hline 6 & 101 & $5.44 \mathrm{p}$ \\
\hline 7 & 110 & $6.08 \mathrm{p}$ \\
\hline 8 & 111 & $15.72 \mathrm{p}$ \\
\hline
\end{tabular}

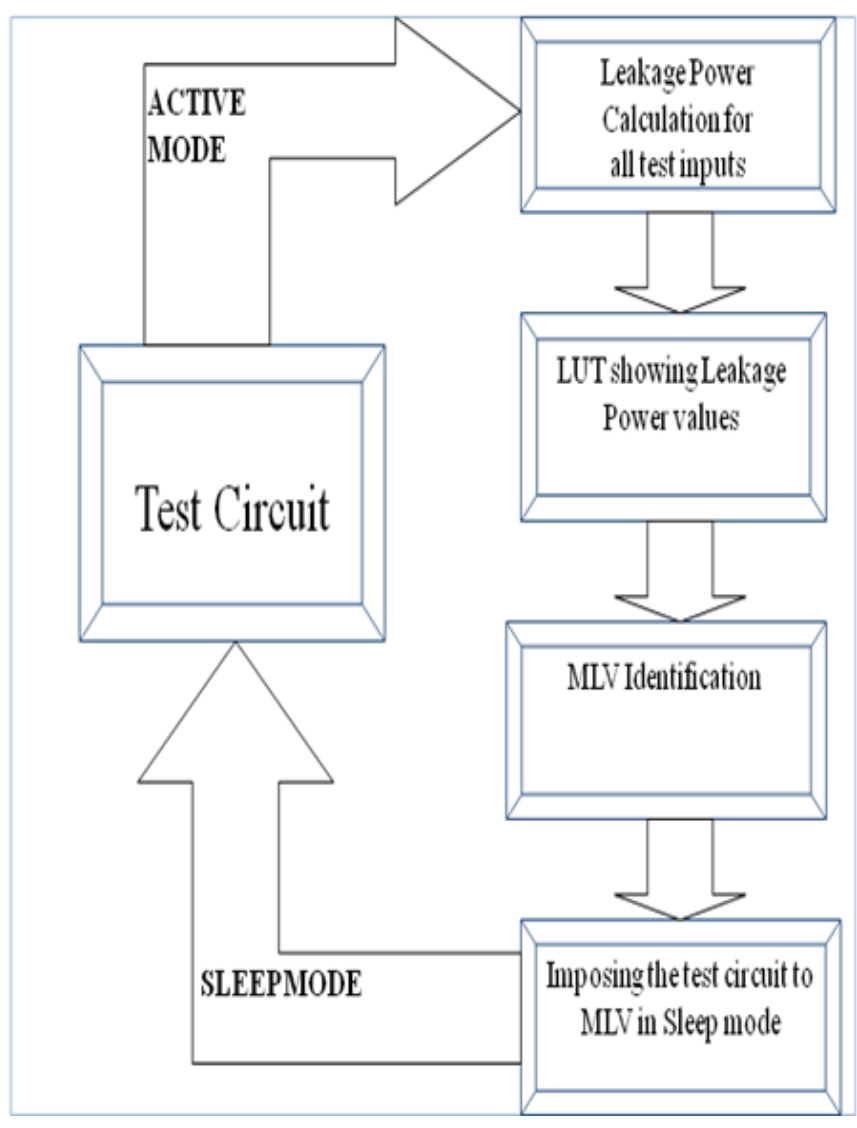

Fig.1. Design Procedure in IVC Approach

\section{ii. Gate Replacement Algorithm}

In Gate Replacement Algorithm, significant leakage reduction can be achieved by identifying Worst Leakage State (WLS) gates which consume more leakage power. These WLS gates are replaced with standard cell library gates as shown in Fig. 2. Gate Replacement Algorithm uses the concept of stacking effect to reduce leakage power. Gate Replacement Algorithm can be presented with the following steps:

1. $\operatorname{Gate}_{\text {new }}(X, 0)=\operatorname{Gate}(X)$ for sleep $=0$ in active mode

2. $\operatorname{Gate}_{\text {new }}(\mathrm{X}, 1) \neq \operatorname{Gate}(\mathrm{X})$ for sleep $=1$ in standby mode

3. Leakage $\left\{\operatorname{Gate}_{\text {new }}(\mathrm{X}, 1)\right\}<$ leakage $\{\operatorname{Gate}(\mathrm{X})\}$ in standby mode
Where ' $\mathrm{X}$ ' is the gate input and sleep is an extra input added in Gate Replacement while converting from the two-input NAND gate to three-input NAND gate. As shown in Fig. 2,

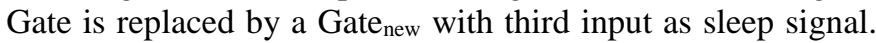
When the transistors in a circuit are making transitions from $\mathrm{ON}$ to $\mathrm{OFF}$ or OFF to $\mathrm{ON}$, the circuit is said to be in active mode. Further, when the transistors are in idle state by not making any transitions, the circuit is under standby mode. Indeed, the gate's functionality will not change during active mode with the additional input connected. However, in standby mode this Gate replacement reduces leakage power.

From TABLE. 1 and TABLE.2, it can be observed that, this Gate replacement reduces leakage power in standby mode from $10.48 \mathrm{pW}$ (for 11 state) of the two-input gate to $6.08 \mathrm{pW}$ (for 110 state) of the three-input gate. This results in a leakage power savings up to $41.98 \%$. Nikhil jayakumar et al. [17] proposed simultaneous Input Vector Control and Circuit modification. A combined Gate Replacement and Input Vector Control approach is presented by Lin yaun and Gang $\mathrm{Qu}$ [18] which outperforms pure IVC in reducing leakage. Authors in [19] explore Direct Gate Replacement (DGR) and Divide and Conquer Based Gate Replacement (DCBGR) algorithms. They investigated that DGR and DCBGR algorithms are capable of achieving better results than pure IVC. In [20] Gate Replacement is discussed in a slack-aware manner to reduce leakage with zero penalty. Surabhi singh et al. [21] proposed a Modified Gate Replacement Algorithm in 2013. Algorithm gives a brief concept of leaving those Worst Leakage State (WLS) gates which increases overall leakage after Gate Replacement.

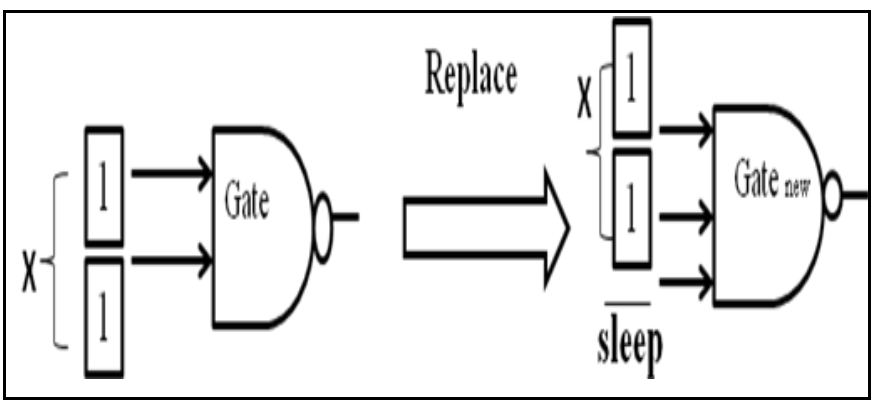

Fig.2.Gate Replacement of a NAND gate

\section{Combined Gate Replacement and Transmission Gate Based Logic Insertion Algorithm}

This section introduces proposed technique called Combined Gate Replacement and Transmission Gate Based Logic Insertion Algorithm. The proposed technique combines the effect of Gate Replacement and inserting a Transmission Gate Based Logic to inputs of the Worst Leakage State (WLS) gates to have better leakage reduction. Based on the requirement, for the same gate both Transmission gate insertions and Gate Replacements are carried out. With the Transmission gate insertions the best input states for the logic gates are imposed and at the same time Gate Replacement introduces pull down stack effect. The stacking effect due to Gate Replacement increases effective resistance between supply rails and this in turn reduces leakage current. The 
concept of the proposed approach is illustrated below in Fig.3.

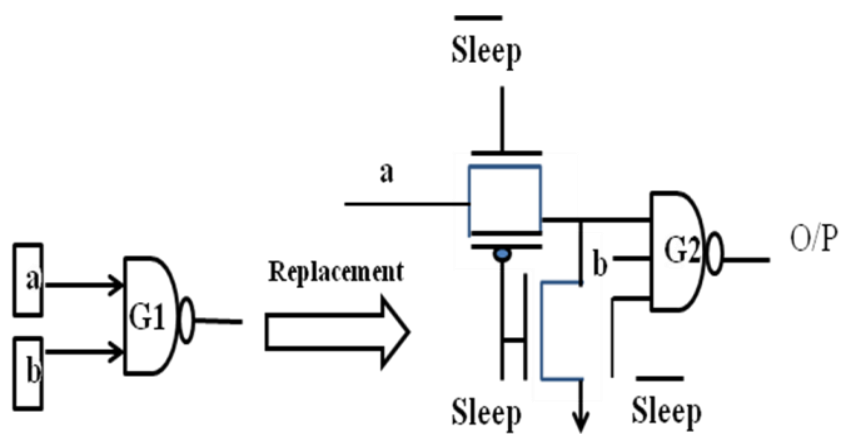

Fig.3.Proposed Technique for Leakage reduction

In the Fig.3 gate G2 functions like a gate G1 when sleep= ' 0 ' during active mode. In standby mode sleep $=$ ' 1 ' makes Transmission Gate to be turned OFF. This drives the first input of $\mathrm{G} 2$ to the ground through a pull down NMOS transistor. This inserts logic ' 0 ' to the first input of gate G2. Second input b of gate G2 is assumed to be ' 1 '. Third input of gate G2 is connected with sleep due to Gate Replacement effect, which is at logic ' 0 ' during standby mode. Hence, gate G2 maintains an input pattern of "010" during standby mode consuming a leakage power of $92.79 \mathrm{fW}$. However, gate G2 with only Gate Replacement technique is consuming $6.08 \mathrm{pW}$ (' 110 ' state in TABLE.2). Hence, the proposed algorithm is very effective and has given up to $98.48 \%$ savings in leakage power compared to Gate Replacement alone and 99\% savings compared to the basic two-input NAND gate G1. Algorithm of the proposed technique is shown in Fig.4.

Algorithm: Combined Gate Replacement and Transmission Gate Based Logic Insertion Algorithm

Input: $\left\{\right.$ gate $_{1}$, gate $_{2} \ldots$ Gate $\left._{n}\right\}, \mathrm{n}$ is the number of gates in a circuit

Find internal node values of all gate inputs.

Forj $=1$ to $n$ do

If gate $_{j}$ is at minimum leakage state

\section{then}

No replacement and insertions for gate ${ }_{j}$

else

do

Gate Replacement with Transmission Gate based Logic

Insertion for gate ${ }_{j}$.

Update new values of Leakage and Delay

end

end

\section{Fig.4. Algorithm of proposed technique}

Input of the algorithm is ' $n$ ' which represents the number of gates in a test circuit. Internal node values at all gate inputs are measured to check for any requirement of replacements and insertions. If any gate is already at a minimum leakage state satisfying the requirement, there is no need of gate replacements and logic insertions. Algorithm will go to next consecutive gates to search for worst leakage and second worst leakage gates. If any gate is identified to be at WLS, Gate Replacement with Transmission Gate based Logic Insertion is applied. After the insertion, leakage and delay values are updated. Based on "sleep" value, the test circuit may be in active mode or in standby mode. Circuit functionality is maintained during active mode and in standby mode all gates are imposed to their best leakage states to consume minimum leakage in the circuit. Proposed algorithm is analyzed with an example of $\mathrm{C} 17$ benchmark circuit shown in Fig.5.

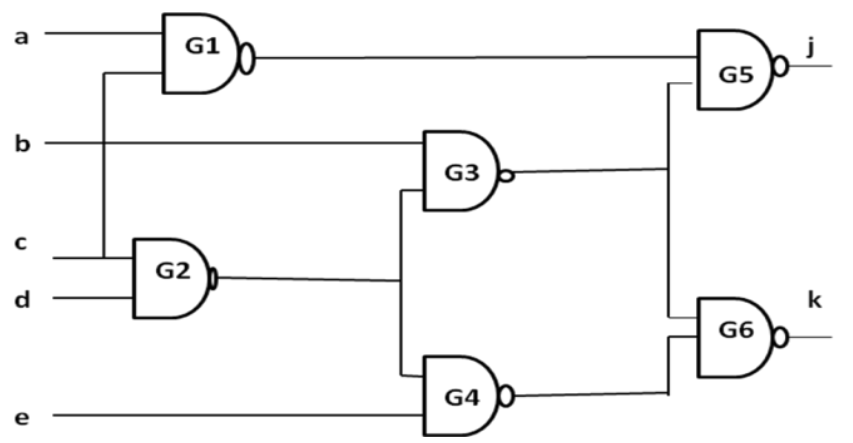

(a)

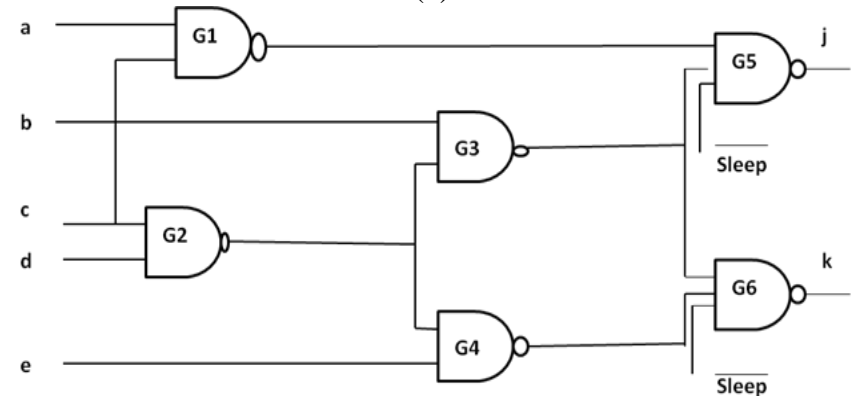

(b)

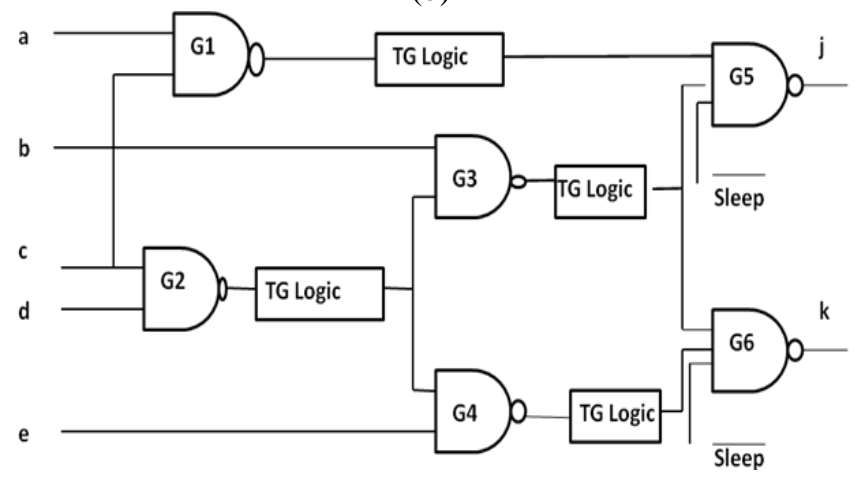

(c)

Fig.5. Test circuit considered as an example (a) $\mathrm{C17}$ Benchmark circuit (b)Gate Replacement applied to C17 Benchmark (c)Proposed Algorithm applied to C17 Benchmark

Fig.5 (a) shows a $\mathrm{C} 17$ benchmark circuit. As shown in Fig.5 (a) "00010" is applied at primary inputs. This input test pattern is found by exhaustive search which gives minimum leakage in the circuit. After this, the proposed algorithm is applied to the test circuit to further reduce leakage. From 
Fig.5 (a) gates G5 and G6 are found to be at the Worst Leakage States (WLS) with "11" as their inputs. Fig.5 (b) illustrates how a Gate Replacement technique is applied to G5 and G6 gates. Consequently, this made inputs of G5 and G6 from " 11 " to " 110 " which has reduced leakage power from $38.01 \mathrm{pW}$ to $19 \mathrm{pW}$. Hence, there is $50.01 \%$ of leakage reduction using Gate Replacement alone. Fig.5(c) depicts the proposed algorithm. This combines the effect of Gate Replacement and transmission Gate based Logic Insertion. The Proposed algorithm makes maximum possible gates in the circuit to be at minimum leakage states. From Fig.5(c), it can be observed that a Transmission gate based logic insertion is applied at the outputs of G1, G2, G3, G4 gates.

The Transmission gate logic at the output of G1, G3, G4 gates makes G5 and G6 gate inputs to be "000" from "110" state. Similarly, Logic insertion at G2 gate output makes inputs of G3 and G4 gates to be at " 00 " from " 10 " and " 01 " sates. Hence, it can be noticed that all the six gates in the circuit are at their minimum leakage states, consuming a power of $15 \mathrm{pW}$.This has given leakage savings of up to $60.53 \%$ compared to the original circuit and up to $21.05 \%$ with respect to Gate Replacement alone. Analysis is presented for a case of C17 benchmark circuit in terms of leakage power savings. However, there is an area overhead with the proposed technique. With six gates in $\mathrm{C} 17$ benchmark, the proposed technique needs additional twelve transistors. This requires $33 \%$ more area consumption. With aggressive scaling towards the nanometer regime, having very high packing densities, the area over ahead caused by the proposed method is not a big issue to accommodate.

\section{Results and Analysis}

Gate replacement technique and Proposed Algorithms are implemented on six different benchmarks of ISCAS'89 to validate the effectiveness. Benchmark circuits are simulated with $180 \mathrm{~nm}$ standard cell library using Hspice Tool. TABLE.3 details simulation results and comparison of the proposed algorithm with Gate replacement in terms of leakage savings. Fig. 6 presents Simulation Results of $\mathrm{C} 17$ benchmark circuit during functional verification using Hspice in active mode. Fig.7 gives measurement of leakage power during standby mode and is calculated to be $15.82 \mathrm{pW}$ for the test case of $\mathrm{C} 17$ and the same is indicated in TABLE.3.

Each WLS gate in a circuit is applied with both Gate Replacement technique and proposed algorithm. In TABLE.3, column 2 presents leakage values of Gate Replacement technique. Leakage power of various benchmark circuits applied with the proposed approach are given in column 3 . Finally, column 4 presents the percentage of leakage improvements of different benchmarks. Fig.8 shows the comparison of benchmark circuits with respect to the percentage of leakage savings. There is an improvement of $16.41 \%$ on average in leakage reduction with the proposed approach compared to Gate replacement. Proposed algorithm could effectively reduce leakage power compared to other state-of-art algorithms like Gate Replacement. As the proposed method requires more transistors to be inserted, there is an increase of area overhead and complexity for larger circuits. With the increasing popularity of a battery powered and portable electronics, reducing power consumption is a significant issue in VLSI industry. Even in applications like sensor networks, wearable electronic devices and in bio medical devices, power consumption is the ultimate issue to be solved than other metrics. With power reduction, longer battery life and the reduced system weight resulting in smaller battery packs is possible. Along with this, reducing the power consumption increases chip reliability also by decreasing heat dissipation. So, in applications where reducing power consumption is the important metric to be considered, the proposed method can be adopted even though complexity increases.

Fig.9 shows the percentage of leakage savings of all benchmarks tested. By exhaustive search of the complete circuit, WLS gates and second WLS gates are identified. These gates are applied with Gate Replacement and then Transmission Gate based logic insertion to have all gates with best leakage inputs. From the above graph, it is found that S641 benchmark has less percentage of leakage savings. This is due to a fact that, this benchmark has maximum gates already at their best leakage states. Thus, the percentage of leakage reduction achieved is less in this case. C432 has more percentage of leakage savings among all benchmarks, because of maximum gates at their worst leakage condition. Therefore, the more number of replacements and insertions are required in this case and proposed algorithm could achieve higher leakage savings.

TABLE.3. Comparison between Gate Replacement and Proposed Algorithms

\begin{tabular}{|c|c|c|c|}
\hline \multirow{2}{*}{$\begin{array}{c}\text { Benchmark } \\
\text { Circuit }\end{array}$} & $\begin{array}{c}\text { Gate } \\
\text { Replacement } \\
\text { Algorithm }\end{array}$ & $\begin{array}{c}\text { Proposed } \\
\text { Algorithm }\end{array}$ & $\begin{array}{c}\text { Improvement in } \\
\text { Leakage } \\
\text { Reduction (\%) }\end{array}$ \\
\hline C17 & $0.019 n$ & $0.015 n$ & 21.05 \\
\hline B02 & $0.247 n$ & $0.217 n$ & 12.14 \\
\hline S27 & $0.143 n$ & $0.122 n$ & 14.68 \\
\hline S420 & $1.740 n$ & $1.490 n$ & 14.36 \\
\hline C432 & $1.040 n$ & $0.730 n$ & 29.80 \\
\hline S641 & $2.950 n$ & $2.760 n$ & 6.44 \\
\hline
\end{tabular}

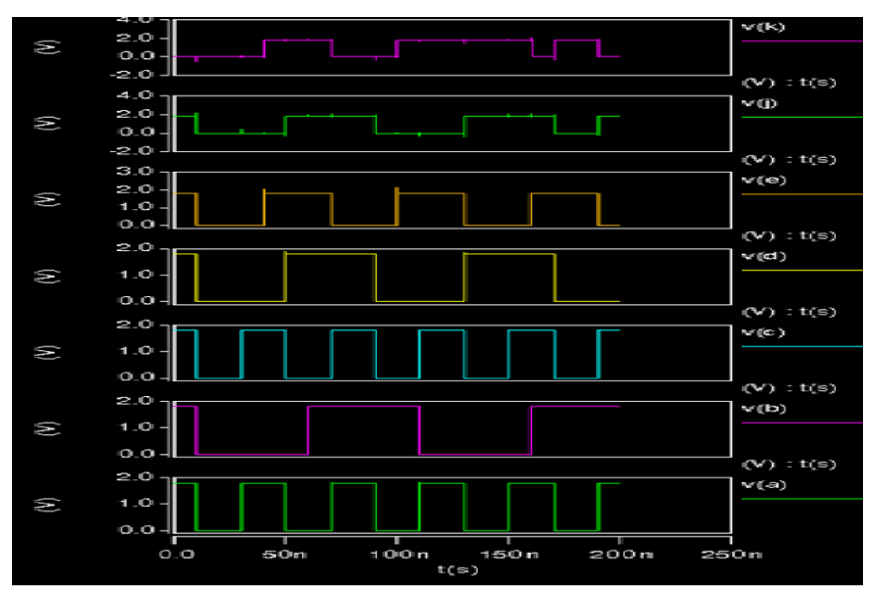

Fig.6. Simulation Results of C17 Benchmark applied with Proposed Technique 


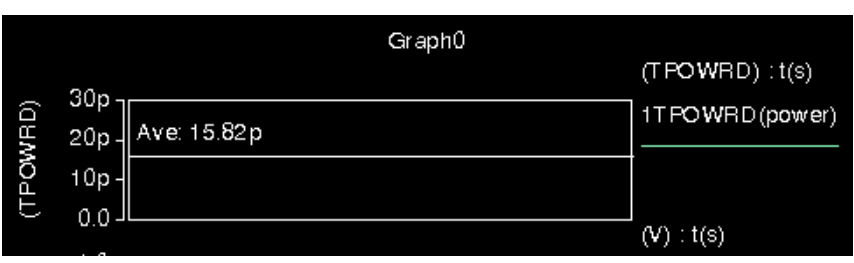

Fig.7. Leakage Power Measurement of C17 Benchmark applied with Proposed Technique

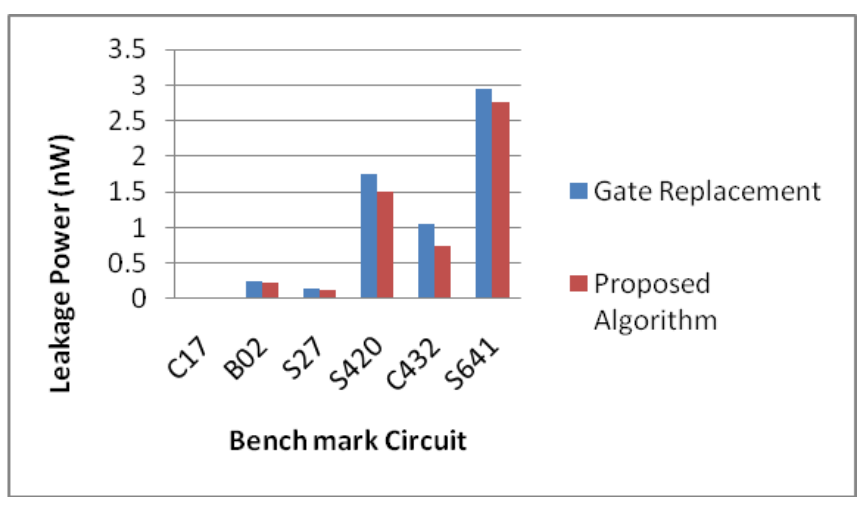

Fig.8.Comparison between Proposed Technique and Gate Replacement technique

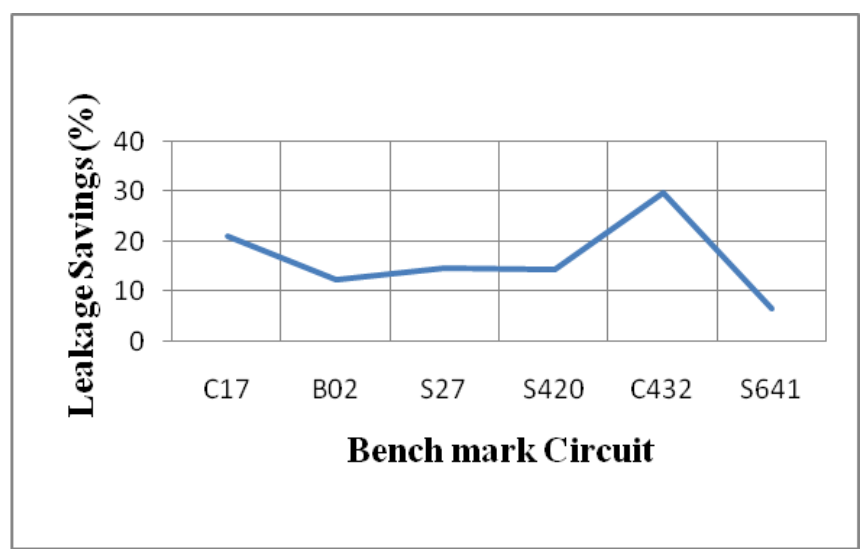

Fig.9. Percentage of Leakage savings

\section{Conclusion}

Advancing trends of technology has led to an increase of leakage currents in MOS transistors. In this paper, a novel Gate Replacement with Transmission Gate based Logic Insertion Algorithm is proposed to reduce such leakages. Gate Replacement along with Transmission gate based insertion is performed for each WLS gate in a circuit to have better leakage reductions. Indeed, this makes maximum possible gates in a circuit to be at their minimum leakage states. Simulation results explores that proposed algorithm has achieved leakage savings up to $16.41 \%$ on average compared to other state-of-art algorithms like Gate Replacement. In future proposed algorithm can be used to mitigate the NBTI effect.

\section{References}

[1] Kaushik Roy; Saibal Mukhopadhyay;Hamid Mahmoodi-Meimand.Leakage Current Mechanisms and Leakage Reduction Techniques in Deep-Sub micrometer CMOS Circuits.PROCEEDINGS OF THE IEEE.2003, VOL. 91, NO. 2.

[2] Se Hun Kim; Vincent J. Moone. Sleepy keeper:a New Approach to Low-leakage Power VLSI Design. VLSI SOC conference. 2006, PP. 367-372.

[3] Jun Cheol Park; Vincent J.Moone. Sleepy Stack Leakage Reduction. IEEE TRANSACTIONS ON VERY LARGE SCALE INTEGRATION(VLSI)SYSTEMS.2006, vol.14, No.11, pp. 1250-1263.

[4] Farzan Fallah; Massoud Pedram. Standby and Active Leakage Current Control and Minimization in CMOS VLSI Circuits. IEICE Transactions.2005, pp.509-519.

[5] Afsin abdollahi;Farzan fallah;Massoud pedram. Leakage current reduction in CMOS vlsi circuits by input vector control. IEEE transactions on very large scale integration (vlsi) systems.2004, vol. 12, no. 2.

[6] Lin Yaun; gang Qu. Enhanced Leakage Reduction Technique by Gate Replacement. Proceedings of 42nd Design Automation Conference.2005, pp. 4750.

[7] Lei Cheng; Deming Chen; Martinwong,D.F. A Fast Simultaneous Input Vector Generation and Gate Replacement Algorithm for Leakage Power Reduction. ACM Transactions on Design automation of Electronic systems.2008, vol.13, No.2.

[8] Chin-Hung Lin; Ing-Chao Lin; Kuan-Hui Li. TGbased Technique for NBTI Degradation and Leakage Optimization. IEEE international conference.2011.

[9] Halter,J.P.; Najm,F.N. A gate-level leakage power reduction method for ultra-low-power CMOS circuits. Proceedings of Custom Integrated Circuits Conference. 1997, pp. 475-478.

[10] Ye, et al. A new technique for standby leakage reduction in highperformance circuits. Symposium on VLSI Circuits Digest of Technical Papers.1998, pp. 40-41.

[11] Zhanping chen;Liqiong Wei;Mark johnson;Kaushik Roy. Estimation of standby leakage power in CMOS circuits considering Accurate modeling of Transistor stacks. international symposium on Low power electronics and design, ISLPED.1998, pp.239-244.

[12] ABDOLLAHI, A.; FALLAH, F.;MASSOUD. Runtime mechanisms for leakage current reduction in CMOS VLSI circuits.Proceedings of the International Symposium on Low Power Electronics and Design.2002,pp. 213-218.

[13] Abdollahi,A; et al. Leakage current reduction in CMOS VLSI circuits by input vector control. IEEE Trans. Very Large Scale Integration Systems.2004,vol. 12, pp. 140-154.

[14] Abdoul Rjoub;Almotasem Belleah Alajlouni; Hassan Almanasrah. A Fast input Vector Control Approach for subthreshold Leakage power reduction. IEEE 
International Journal of Applied Engineering Research ISSN 0973-4562 Volume 10, Number 21 (2015) pp 42610-42615

(C) Research India Publications. https://dx.doi.org/10.37622/IJAER/10.21.2015.42610-42615

electro technical international conference,MELECON.2012.

[15] Zhanping chen;Liqiong Wei;Mark johnson; Kaushik Roy. Estimation of standby leakage power in CMOS circuits considering Accurate modeling of Transistor stacks.ACM transactions, international symposium on Low power electronics and design, ISLPED.1998, pp.239-244.

[16] LeelaRani,V; Madhavi Latha,M. Implementation of genetic algorithm for minimum leakage vector in input vector control approach. IEEE conference,SPACES. 2015.

[17] Nikhil Jayakumar; Sunil Khatri,P. An Algorithm to Minimize Leakage through Simultaneous Input Vector Control and Circuit Modification. IEEE Design Automation conference,DATE07.2007.

[18] Lin Yuan; Gang Qu. A combined gate replacement and input vector control approach for leakage current reduction. IEEE transactions on very large scale integration (vlsi) systems.2006, vol. 14, no. 2.

[19] Wang,Y; et al. Leakage Power and Circuit Aging Cooptimization by Gate Replacement Techniques. IEEE Transactions on Very Large Scale Integration (VLSI) Systems.2010, vol. PP, pp. 1-14.

[20] Nikhil Jayakumar; Sunil Khatri,P. A Simultaneous Input Vector Control and Circuit Modification Technique to Reduce Leakage with Zero Delay Penalty. ACM Transactions on Design Automation of Electronic Systems.2010, Vol.16, no.1,pp.1-20.

[21] Surabhi singh; Baljit Kaur;S.Das gupta. A Modified gate Replacement Algorithm for Leakage Reduction UsingDual-Tox in CMOS VLSI Circuits. VDAT 2013.CCIS 382,2013, pp.146-152. 